\section{Dyspnoe in der Notauf- nahme: häufig und nicht immer harmlos}

Hale ZE et al. Causes of Shortness of Breath in the Acute Patient: A National Study. Acad Emerg Med 2018; doi:10.1111/acem.13448

Dyspnoe beschreibt das subjektive Gefühl, nicht ausreichend Luft zu bekommen und stellt ein häufiges Symptom in der Notaufnahme dar. Da sich hinter dieser Symptomatik die verschiedensten Krankheitsbilder verbergen können und die Differenzialdiagnose mitunter schwierig sein kann, haben Hale und Kollegen den Zusammenhang zwischen klinischer Symptomatik in der Notaufnahme und späterer Diagnose genauer unter die Lupe genommen.

Dyspnoe gehört zu den häufigsten Gründen für das Aufsuchen einer Notaufnahme, in den Vereinigten Staaten von Amerika gibt es pro Jahr etwa 3,4 Millionen Fälle. Obgleich bereits Studierende lange Listen möglicher Differenzialdiagnosen zu Rate ziehen können, bleibt insbesondere die Einschätzung der Ernsthaftigkeit der Symptomatik jedes Mal wieder eine große Herausforderung.

Vor diesem Hintergrund haben Hale und Kollegen mit Hilfe einer großen Datenbankanalyse untersucht, wie die Symptomatik und klinische Parameter von Patienten mit Dispnoe in der Notaufnahme mit der späteren Diagnose und einer eventuellen intensivmedizinischen Behandlung zusammenhängen. Sie griffen dafür auf die Daten der National Hospitality Medical Care Survey Datenbank für den Zeitraum zwischen 2005 und 2014 zurück.

In dieser Datenbank werden regelhaft für jedes Jahr sämtliche Merkmale von Besuchen US-amerikanischer Notaufnahmen registriert. Stellvertretend für etwa 42 Millionen Besucher einer Notaufnahme sammelten die Forscher die Daten von 10170 Fällen. Symptomatik und klinische Parameter wie Sauerstoffsättigung und Atemfrequenz wurden je nach Altersgruppe zusammengestellt. Darü-

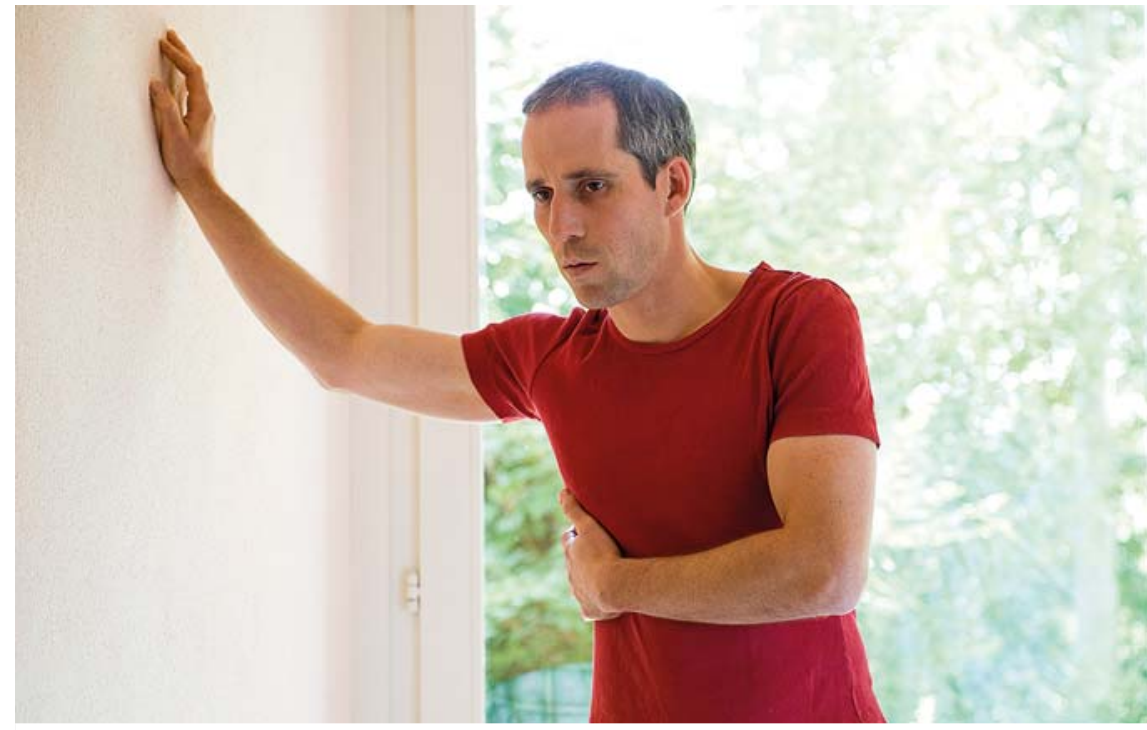

- Keine oder nicht ausreichend Luft zu bekommen vermittelt dem Patienten stets starke Angst. Quelle: Paavo Blåfield/Thieme Verlagsgruppe

ber hinaus stellten die Studienautoren die entsprechenden Entlassungsdiagnosen und die Häufigkeit eventueller stationärer und intensivmedizinischer Behandlungen fest.

\section{Ergebnisse}

Die meisten Patienten mit Dispnoe als führendem Symptom in der Notaufnahme waren zwischen 45 und 64 Jahre alt (31,6\%). Über die Hälfte aller Patienten konnten noch am Aufnahmetag direkt aus der Notaufnahme wieder in das häusliche Umfeld entlassen werden (57,5\%), $8,1 \%$ aller eingeschlossenen Fälle benötigten eine intensivmedizinische Behandlung.

In der Altersgruppe zwischen 18 und 44 Jahren stellte die akute Exazerbation eines Asthma bronchiale mit 14,8\% die häufigste Verdachtsdiagnose dar. Bei älteren Patienten zwischen 45 und 64 Jahren sowie zwischen 65 und 79 Jahren stellten die Forscher am häufigsten die Diagnose einer chronisch obstruktiven Lungenerkrankung bzw. einer chronisch obstruktiven Bronchitis (11,1\%). In der Altersgruppe ab 80 Jahren war eine dekompensierte Herzinsuffizienz mit $12,4 \%$ die häufigste Ursache der subjektiven Atemnot.
Zur Überraschung der Forscher konnten bei 44,6\% aller Patienten die Vitalparameter als normal eingestuft werden. Bei Patienten, die stationär aufgenommen und intensivmedizinisch behandelt werden mussten, kamen eine erhöhte Atemfrequenz oder eine erniedrigte Sauerstoffsättigung dagegen häufiger vor. Die Forscher beurteilten ihre Ergebnisse im Diskussionsteil als nützlich für die Einschätzung von Vortest-Wahrscheinlichkeiten als Hilfsmittel im Prozess der Differenzialdiagnostik.

\section{FAZIT}

Dyspnoe zählt zu den häufigsten Symptomen in einer Notaufnahme, die Differenzialdiagnose ist oftmals eine große Herausforderung. Während bei jüngeren Patienten Asthma bronchiale die häufigste Ursache darstellte, litten ältere Patienten überwiegend an einer chronisch obstruktiven Bronchitis oder einer Herzinsuffizienz. Obgleich über die Hälfte der untersuchten Patienten direkt nach Hause entlassen werden konnten, kann sich hinter subjektiver Atemnot ebenso eine ernsthafte Erkrankung verbergen.

Dipl.-Psych. Annika Simon, Hannover 